\title{
EL DESARROLLO SOCIAL SOSTENIBLE: UN ANÁLISIS DESDE LA GESTIÓN ENFOCADA EN LA SOCIOFORMACIÓN
}

\author{
SUSTAINABLE SOCIAL DEVELOPMENT: AN ANALYSIS \\ FROM MANAGEMENT FOCUSED ON SOCIOFORMATION
}

\author{
Alexandra Soledad Freire Ruíz ${ }^{1}$ \\ Recibido: 2020-08- 20 / Revisado: 2020-10-01 / Aceptado: 2020-11-01 / Publicado: 2021-01-01
}

Forma sugerida de citar: Feire-Ruiz, A. S. (2021). El desarrollo social sostenible: un análisis desde la gestión enfocada en la soicoformación. Retos de la Ciencia. 5(10), pp. 27-37. https://doi.org/10.53877/rc.5.10.20210101.03

\section{RESUMEN}

Actualmente no existe un equilibrio entre el progreso social, económico y medioambiental debido al pensamiento individualista de las nuevas generaciones, por tanto, es indispensable trazar una ruta para potencializar el progreso social que busque el equilibrio para el desarrollo social sostenible que encuentre el bienestar para todos. Las metas deben enfocarse en la implicación activa de las personas, de las empresas, de los distintos actores que nos rodean como instituciones de educación superior. Lo que permitirá afrontar grandes desafíos y preparar de forma eficiente a los futuros profesionales que se están preparando en las universidades con el fin de lograr un verdadero desarrollo social sostenible de forma transversal en toda la gestión universitaria.

Palabras clave: desarrollo, gestión, social, socioformación, sostenible.

\begin{abstract}
Currently there is no balance between social, economic and environmental progress due to the individualistic thinking of the new generations, therefore, it is essential to draw a route to potentiate social progress that seeks the balance for sustainable social development that finds well-being for all. The goals should focus on the active involvement of people, companies, and the different actors that surround us as higher education institutions. This will allow facing great challenges and efficiently preparing future professionals who are preparing in universities in order to achieve true sustainable social development in a transversal way throughout university management.
\end{abstract}

Keywords: development, management, social, socioformation, sustainable.

\footnotetext{
${ }^{1}$ Magister en Gestion Empresarial basada en Metodos Cuantitativos. Docente en la Carrera de Aministración de Empresas de la Universidad Tecnológica Indoamérica. Ecuador. Email: alexandrafreire@uti.edu.ec / ORCID: https://orcid.org/0000-0002-1889-8176
} 


\section{INTRODUCCIÓN}

La globalización, a finales del siglo XX, estimuló la competencia organizacional, obligando a las empresas a ser más flexibles, automatizadas y creativas en su quehacer diario. Esta dinámica trajo cambios innovadores, tales como, una nueva visión estratégica y el desarrollo de habilidades con compromiso social (Fernandez y D’Addosio Serna, 2013). En ese contexto, nuestro país se ha integrado a la corriente mundial en pro del Desarrollo Social Sostenible, hacia una concienciación de participación en las comunidades, al compromiso con la calidad de vida de las personas, porque el mundo, debido a esa globalización, se ha convertido en interdependiente e integrado. Por tanto, se hace imprescindible que las organizaciones universitarias asuman nuevas variables relacionadas con los temas sociales, para crear un compromiso positivo dirigido al desarrollo social y a la construcción de un nuevo mundo para las futuras generaciones (Fernandez de Pelekais y D’Addosio, 2013), capaz de vivir en una sociedad sostenible, libre de discriminaciones que incentive proyectos, soluciones y potencialice la calidad de vida.

La gerencia académica al estar conformada por una correlación de políticas, estrategias y actividades está llamada a ser una herramienta para lograr la transformación de las Universidades en aras de orientar el proceso educativo hacia el desarrollo social sostenible. En ese sentido, esta investigación analizó el desarrollo social sostenible como resultado de la gestión académica la universidad Indoamérica de gestión privada, donde el Desarrollo Social Sostenible, está referido a las necesidades básicas de la población, mejoramiento de su calidad de vida y la gerencia académica; al ser las universidades, instituciones clave para el diseño de proyectos que promuevan dicho desarrollo (Fernandez y D’Addosio, 2013).

De esta forma, (Kruger, 2006) señala que el término sociedad del conocimiento ocupa un lugar estelar en la discusión actual, pues resume las transformaciones sociales que se están produciendo en la sociedad moderna y sirve también para el análisis de estas transformaciones. Al mismo tiempo, ofrece una visión del futuro para guiar normativamente las acciones políticas y es definida como el conjunto de comunidades que trabajan de manera colaborativa en la resolución de sus problemáticas, en donde la utilización de las nuevas tecnologías es de vital importancia para la generación y aplicación del conocimiento al momento de afrontar los retos y necesidades más apremiantes de la población, como lo es el mejoramiento de la convivencia, la calidad de vida, el desarrollo sostenible, por mencionar algunas (Tobón, 2015)

En este nuevo entorno, la sociedad del conocimiento induce desafíos sustantivos a los sistemas e instituciones educativas, pues deben transformarse para formar a las personas que construyan la sociedad del conocimiento, asumiendo nuevos roles y acciones (Rodríguez, 2009). De ahí que la innovación de las prácticas educativas sea un reto clave en la comunidad académica y educativa, pues es necesaria la realización de cierto tipo de prácticas para generar los cambios, tales como la participación colaborativa de todos los miembros de la comunidad educativa, la revisión de las necesidades del contexto y la generación de modelos educativos interdisciplinarios, entre otras (Díaz-Barriga, 2010)

Buscando asumir estos cambios, se ha experimentado con la puesta en práctica de diversos enfoques y modelos educativos, siendo la educación centrada en la sociedad del conocimiento una respuesta actual que están siguiendo los sistemas 
educativos en América Latina. Con ello se busca el desarrollo de las competencias, mismas que se entienden como actuaciones para realizar actividades y resolver problemas buscando el logro de una meta y considerando el contexto con articulación de conocimientos, habilidades, actitudes y valores (Zabalza, 2007). En lo que concierne al abordaje de una educación por competencias por parte de los sistemas e instituciones educativas, generalmente se observa que se quedan en dos extremos, que son: el ideal de formar personas competentes para afrontar los retos de la vida, pero sin estrategias prácticas, o el énfasis en aspectos metodológicos, pero sin un direccionamiento claro.

Como reparar años y años de consumismo desmedido, de daños al ambiente, de falta o carencia de valores, sobre todo la falta de compromiso de los grupos sociales y de las empresas que existen en el mundo. El que se dirijan investigaciones sobre los problemas que aquejan al planeta motiva para hacer este proceso realidad y aplicarlos desde las instituciones iniciando con el diseño y aplicación de proyectos con problemáticas del contexto local de los estudiantes (Narváez-Santos, 2018).

Es importante, necesario e inmediato aplicar estrategias que generen el trabajo colaborativo entre la comunidad educativa; comenzar y aplicar el "proyecto ético de vida", para priorizar, afianzar y desarrollar los valores humanos que tanto hacen falta cultivar en todos y así comprender lo importante que es estar comprometidos a contribuir en el desarrollo social no pensando en el bien individual sino buscando el bien común; que no solo mejoren las condiciones si no que puedan sostenerse a futuro (Narváez-Santos, 2018).

El desarrollo social sostenible tiene como finalidad superar problemas no solo con soluciones inmediatas que sean concretas que generen un equilibrio social y ambiental, sino también cuenta con que haya un reparto equilibrado que cubran las necesidades actuales y las futuras, priorizar en proyectos dirigidos a mejorar el ambiente, se hace imperativo para asegurar el fortalecimiento y duración de los recursos naturales diseñando estrategias definitivas y sostenibles que se pueden realizar si todos contribuimos. El desarrollo social sostenible y la socioformación están de la mano decididos y comprometidos a buscar el cambio en la sociedad iniciando en las instituciones educativas y mostrando soluciones concretas que hagan la diferencia (Narváez-Santos, 2018).

\section{DESARROLLO}

El desarrollo social sostenible es un término que hace referencia al conjunto de obligaciones y compromisos, tanto nacionales como internacionales, derivados del impacto que la actividad humana produce en el ámbito social, laboral, medioambiental, así como de los derechos humanos en todo el planeta. Este desarrollo social, el cual abarca diferentes aspectos externos e internos de las comunidades, obedece al derecho de las personas a vivir en una sociedad más equilibrada, comprometida y justa (Fernandez y D'Addosio Serna, 2013).

Al respecto, el documento denominado Decenio de las Naciones Unidas de la Educación para el Desarrollo Sostenible (UNESCO, 2020), instituye que el desarrollo sostenible, como concepto en constante evolución, es la voluntad de mejorar la calidad de vida de todos, incluida la de las futuras generaciones, mediante la conciliación del crecimiento económico, el desarrollo social y la protección del medio ambiente. 
En ese orden de ideas, en la Estrategia Mundial para la Conservación de la Naturaleza (Fernández y Gutiérrez, 2013) donde se evidencia la necesidad de acuñar el término, se estableció como desarrollo sostenible aquel que satisface las necesidades del presente sin comprometer la capacidad de las generaciones futuras para satisfacer sus propias necesidades.

A su vez, en el documento Panorama Social de América Latina (CEPAL, 2010)emanado de la Comisión Económica para América Latina y el Caribe, se plasma la integralidad del desarrollo con perspectiva de derechos, cuyo dinamismo tenga efectos virtuosos en el empleo e inclusión social, un desarrollo productivo que promueva una mayor convergencia entre sectores y actores del mundo del trabajo con énfasis en conocimiento e innovación, articulación de territorios reduciendo brechas en niveles de bienestar, enfoque de derechos sociales, promoción del empleo decente.

Respecto al desarrollo social sostenible (DSS), (Salud, 2013), indica que incluye una serie de aspectos que van desde tangibles y básicos (agua potable, alimentos saludables, medicamentos y vivienda) hasta aspectos menos tangibles (educación, empleo, equidad y justicia). A su vez, (Hernández, 2011) refiere que se puede considerar como uno de los tres enfoques tipo sobre el desarrollo, junto al enfoque economicista y el enfoque a escala humana, destacando que el objeto y objetivo de este enfoque es el conservar la vida con todo lo que ella implica. Específicamente, refiere que el DSS se compone del pilar económico, social y de protección del medio ambiente, y que consiste en un sistema de equilibrios entre lo social, ambiental y económico, cuyo resultado debe ser la solidaridad. Por lo anterior delimita las cuatro dimensiones por las cuales estaría constituido, siendo los valores éticos de la sociedad, capacidad de asociatividad, grado de confianza entre sus miembros y conciencia cívica.

Una vez comprendido y asumido el trabajo de la socioformación se empata el acto de aplicar el principio de Desarrollo sostenible como lo aborda (Lozano, 2018), quien afirma que debe ser observado desde una aproximación sistémica basada en considerar el binomio Sociedad-Naturaleza como dos sistemas que constituyen uno mayor. Por una parte, los seres humanos organizados, actuando en sociedad como un sistema social, y, por otra parte, la naturaleza como conjunto de procesos auto de un sistema natural, los cuales constituyen el medio ambiente que soporta el desarrollo humano. Con ello, se generará lazos que permitan caminar, formar y fortalecer conceptos planteados para culminar avances dentro de la socioformación.

Las nuevas concepciones en torno al desarrollo social sostenible son: 1) el enfoque socioformativo como una alternativa Latinoamericana busca el desarrollo socioeconómico con formación integral, sustentabilidad ambiental e inclusión social a partir de la necesidad de educar conscientemente a las nuevas generaciones para un futuro mejor. Para ello, se demanda cambios de actitud perennes en el comportamiento de los ciudadanos y así lograr niveles cada vez mayores de calidad de vida con logros en la sustentabilidad ambiental; 2) implica una acción sistemática con una perspectiva compartida orientada a la transformación social compleja y constante con el ecosistema y,

3) tiene una perspectiva inter y transdisciplinaria y con una actitud proclive a la sistematización y ordenamiento metodológico de las prácticas de participación social de manera ética, colaborativa y de gestión por proyectos formativos (Coguox, Sandoval y Izquierdo, 2018). 
La enseñanza para la sostenibilidad representa un proceso para formar personas comprometidas con el progreso humano, desarrollo de sociedades del conocimiento, la sostenibilidad del planeta, la inclusión, educación para todos, acceso a la salud, seguridad alimentaria, y erradicación de la pobreza en todas sus formas (Jarrín \& Tola, 2016). Entonces se pretende con este constructo, apegarse, afianzarse a la línea de la socioformación dado que, va en búsqueda de un desarrollo integral de las personas en distintos ámbitos, mediante proyectos socioformativos, con una mirada ética, en un contexto reflexivo continuo y apoyándose en las tecnologías de la información para sentar las bases de una sociedad del conocimiento (Prado, 2018).

El desarrollo social sostenible en la socioformación "busca formar en y para la sociedad mediante la manera de condiciones de vida y el cuidado del ambiente" (Tobón, 2018). Dentro de las modificaciones en prácticas andragógicas en una institución encargada de la educación superior, será pertinente la incorporación de la tecnología, a través de la educación virtual con el respaldo de las instituciones de gobiernos locales (Castro, 2018), con el respaldo de la participación y aceptación ciudadana.

Otras prácticas novedosas para incorporar son: el proyecto ético de vida, habilidades socioemocionales, el pensamiento complejo y el trabajo colaborativo (Tobón, 2018), debido a que es la forma práctica dentro de las metodologías de incorporar la teoría y la práctica del conocimiento a través de la resolución de los problemas de coyuntura.

Como parte de las características claves en la socioformación, se puede mencionar lo siguiente: Orientación en la formación integral, menciona que el enfoque socioformativo, sugiere que la formación que reciba un individuo debe articular saberes, tales como el saber ser, saber hacer, saber conocer y saber convivir, con la intención de permitirle actuar de manera integral en el abordaje de problemas de contexto, sean estos personales o de la sociedad en la que se desenvuelve.Por ello, es que de vital importancia que sea el propio individuo el que emprenda acciones creativas en la solución de sus necesidades y de los problemas que condensa la sociedad del conocimiento a través del establecimiento de un proyecto ético de vida y metacognición (Tobón, Pimienta y García, 2016).

Involucra a diversos actores, debido a que el currículo tradicional está centrado en el docente, como sucede con el currículo academicista, el funcionalista o el conductual. En años recientes, se ha centrado en el estudiante (currículo constructivista). Por el contrario, el currículo socioformativo se enfoca en formar a todos los actores (directores, docentes, estudiantes, familias, organizaciones y comunidad) con los mismos ejes, como, por ejemplo, la resolución de problemas y el trabajo con proyectos colaboraborativos (Martínez, 2017).

Se basa en la mejora continua. El currículo socioformativo se basa en el mejoramiento continuo de las prácticas de aprendizaje, evaluación, mediación, gestión de recursos, articulación con las organizaciones, trabajo con la comunidad y gestión organizacional, con base en la reflexión y el proyecto ético de vida (Martínez, 2017).Por ello, se vuelve prioritario considerar las necesidades que presenta el contexto y así retroalimentar, actualizar y establecer los futuros retos que ha de enfrentar el currículo, mismo que desde el enfoque socioformativo debe ser flexible, ya que no todos los aspectos de la propia gestión curricular deben ser planificados desde el inicio, sino que es mejor irlos construyendo o adaptarlos a medida que se implementan y se detectan las necesidades de los actores en 
formación y las condiciones de vida requeridas para la sociedad del conocimiento (Tobón, 2015).

Se enfoca en el trabajo colaborativo. Desde el enfoque socioformativo, el currículo se construye de manera continua, con base en la colaboración, a medida que los mismos actores se van formando. Es por ello por lo que se integran diferentes actores tanto en la planeación como en la ejecución y el mejoramiento continuo de organizaciones e instituciones sociales (Martínez y Vasquez, 2017). Esto se hace con base en planes de acción acordados, metas comunes y distribución de roles.

Trabajo con base en proyectos formativos. A diferencia del currículo tradicional, que se centra en asignaturas y clases basadas en contenidos, el currículo socioformativo se implementa mediante diferentes estrategias centradas en problemas del contexto, como son los proyectos formativos (Tobón, 2015). Un proyecto formativo es un conjunto articulado de actividades para resolver un problema contextual mediante la colaboración, el pensamiento complejo, la metacognición y el trabajo interdisciplinario para articular diferentes saberes.

Focalización en la solución de problemas del contexto. Desde el enfoque socioformativo, el currículo no debe estar centrado en la transmisión de contenidos, que en muchas ocasiones no tienen una articulación entre sí (Martínez y Vasquez, 2017) por el contrario, la formación debe orientarse en la resolución de problemas contextualizados. Un problema del contexto consiste en un reto de pasar de una situación dada a una situación ideal o esperada mediante el análisis (Hernández, Gutiérrez y Tobón, 2014; 2016).

Es importante mencionar que en la socioformación del conocimiento es una metodología en la cual se puede trabajar con estudiantes a nivel primario, secundario y universitario, incorporando el conocimiento de la teoría y el desarrollo práctico del mismo a través de actividades amigables al ambiente, tomando en consideración la calidad de vida, el talento humano, el currículo y la tecnología en el estudiante, para adquirir su conocimiento (Coguox, Sandoval, y Izquierdo, 2018).

Definitivamente la Socioformación, a través de la sociedad del conocimiento, muestra el camino para reorientar los procesos desde las instituciones educativas y crear en los estudiantes la metacognición con el objeto de desarrollar el pensamiento crítico y la búsqueda de soluciones a los conflictos que se vienen presentando en el entorno. La información se ha quedado guardada y es necesario transformar el entorno y crear ambientes propicios para compartir conocimientos. Desde la socioformación se puede desarrollar proyectos para preservar el medio ambiente y así mejorar la calidad de vida, desarrollar el talento de los estudiantes y encaminar el proyecto de vida (Borrego, 2018).

En los procesos educativos humanistas el enfoque no se centra en la instrucción sino en el aprendizaje, de tal forma que se concibe al alumno como un ente activo de su propio desarrollo. Sus intereses y conceptos previos determinan la manera en que interactúa con su entorno, generando nuevas visiones, conceptos, aptitudes, motivaciones y formas de actuar, por lo que debe aprender a ser persona y ciudadano (Moreno-Hernández y Rivera-Hernández, 2017).

Precisamente, en el concepto de ciudadano radica la gran relevancia para las contribuciones hacia el desarrollo sostenible. Debe reconocerse a la socioformación como una herramienta que permite resolver problemas del entorno, ayudando en el proceso a que estudiantes y facilitadores se desenvuelvan de manera colaborativa y completa, con vidas sólidas y éticas (Niño-Castillo, 2018) 
El mundo exige que el individuo se adapte a sus cambios vertiginosos en la parte educativa, económica, tecnológica, cultural y social, por lo que es necesario que desde la docencia se innoven las prácticas educativas donde el estudiante es el centro y dejar de concentrarse solamente en la transmisión de conocimientos (Ambrosio, 2018). Para tal efecto, la socioformación propone los proyectos formativos, los cuales se definen como un conjunto de actividades articuladas para identificar, interpretar, argumentar y resolver problemas del contexto. Se estimula la formación de estudiantes competentes, emprendedores y éticos al promover el trabajo en colaboración (Tobón, Cardona, Vélez-Ramos y López-Loya, 2015).

Se ha probado que los proyectos formativos favorecen la formación integral de los individuos y promueve cambios positivos en el rol del maestro al convertirlo en un motivador y guía del conocimiento (Arnao, Medina, Calderón, Esquivez y Tello, 2014), teniendo un impacto positivo en ellos mismos, pero, sobre todo, en su comunidad y contexto en general.

Tobón (2018) abunda en la idea de los proyectos formativos argumentando que permiten a los alumnos aprender tal y como sucede en la vida cotidiana; en la forma de resolver problemas; fortalece su motivación y la de los demás actores en su comunidad; contribuye a mejorar sus condiciones de vida; y trascienden su aplicación a otros ámbitos que van más allá de la escuela. Quizá el mejor cambio de conducta se logre al ser ejemplo en los temas torales de la vida y la sociedad: ante la alimentación, ante la violencia, ante la corrupción, etc. Eastmond (2005) dice que la mejor manera de promover cambios es estimulándolos desde la acción, a través del ejemplo, ya que se ha probado que el contacto directo con personas que muestran congruencia entre lo que dicen y lo que hacen tiene un alto impacto entre quienes se están formando. Por si fuera poco, Tahini, Nakayama, Dibrova y Dadykin (2018) lo refuerzan al decir que cuando el estudiante reconoce que sus esfuerzos de aprendizaje le traen resultados en la práctica, se genera un estímulo incondicional para seguir estudiando e incrementa su motivación hacia el aprendizaje.

El modelo educativo de una universidad se define como la visión compartida del tipo de formación que se pretende lograr en todos los actores de la comunidad universitaria (estudiantes, docentes, directivos, egresados y comunidad), integrando la investigación, el emprendimiento y la vinculación con la sociedad. Busca responder a los retos y necesidades de la comunidad, las organizaciones, la ciencia, la tecnología, el ambiente, la calidad de vida, el desarrollo socioeconómico y la sostenibilidad ambiental. Comprende la filosofía, los principios, la epistemología y los lineamientos generales que deben considerarse en todos los ámbitos de la institución, de acuerdo con las funciones sustantivas de las instituciones de educación, en el marco de la ley y la autonomía.

La sociedad está teniendo cambios radicales en todos los ámbitos y en poco tiempo ha pasado de la sociedad industrial a la sociedad de la información, tal y como puede verse en la cultura actual centrada cada vez más en lo digital. Sin embargo, el reto es crear y vivir en la sociedad del conocimiento, de tal manera que se fortalezca la convivencia, el emprendimiento, la calidad de vida, la cultura de la paz, el sentido de la vida, la economía a escala humana y la sostenibilidad ambiental, mediante la gestión y cocreación del conocimiento científico a través de fuentes rigurosas. Esto implica para la Universidad Tecnológica Indoamérica trabajar con las comunidades en el mejoramiento de sus condiciones de vida, con base en la resolución de los problemas prioritarios mediante la autogestión y el empoderamiento, con análisis crítico y sistémico, trabajo colaborativo entre todos los 
ciudadanos, solidaridad, perseverancia y el uso de las tecnologías de la información y la comunicación (Slater, 2014; Stehr, 1994; Tedesco, 1999; Tobón et al., 2015; Viglierchio y Williamson, 2015).

El Modelo Educativo de la Universidad Tecnológica Indoamérica busca proyectarse hacia una educación de excelencia, que permita la acreditación como universidad pertinente en el Ecuador y en el mundo. Para ello se plantean una serie de lineamientos de gestión curricular, docencia, investigación, vinculación con la colectividad, dirección estratégica y evaluación permanente, que tienen como finalidad mejorar el desempeño de todos los actores y procesos de la institución para generar transformaciones en la comunidad que posibiliten mejorar las condiciones de vida, a partir de la integración local, nacional e internacional, considerando los saberes ancestrales pero también generando y buscando la apropiación de los saberes científicos, en articulación sistémica (Indoamérica, 2018).

La universidad debe cumplir sus funciones sustantivas (docencia, investigación, vinculación con la sociedad y gestión administrativa) (Universidad Tecnológica Indoamérica, [UTI], 2018), a partir del desarrollo del talento humano en todos sus actores, articulando la tecnología y buscando ser, en sí misma, una sociedad del conocimiento. Esto significa trabajar de manera continua en mejorar e innovar los diferentes procesos a través del enfoque de la resolución de problemas mediante la colaboración y empleo de las tecnologías de la información y la comunicación, con base en el desarrollo del pensamiento complejo y desde una visión multi, inter y transdisciplinaria con apoyo en la ética y la inclusión.

Para lograr construir la sociedad del conocimiento y desarrollar el talento en los diferentes actores, la Universidad Tecnológica Indoamérica asume el enfoque socioformativo por considerar que es el que mejor se articula con la visión, misión y valores de la universidad. Se trata de un enfoque innovador, de origen latinoamericano, en el cual la Universidad ya lleva trabajando varios años y que se articula plenamente a las actuales políticas de la educación superior del Ecuador establecidas en la Constitución, la Ley Orgánica de Educación Superior (LOES), los reglamentos de esta ley y los lineamientos del régimen académico para la elaboración de las carreras y programas, en cuanto busca el desarrollo social sostenible con base en proyectos colaborativos mediante la transdisciplinariedad. (UTI, 2018) Este enfoque está basado en la investigación, se aplica en diferentes países y está generando cambios sustanciales en la educación superior al fortalecer la actuación ética y buscar productos concretos en la resolución de los problemas que mejoren las condiciones de vida, considerando la diversidad cultural y los procesos de inclusión.

La construcción del modelo educativo ha considerado las tendencias internacionales e innovaciones que se están dando en la educación superior, a partir de un proceso de investigación riguroso, basado en la revisión de la literatura académica como también en el análisis de experiencias en el Ecuador y Latinoamérica (UTI, 2018). Así mismo, se consideraron los aprendizajes logrados en la implementación del modelo anterior bajo el mismo enfoque socioformativo, pero centrado solo en la docencia de grado. Ahora se busca un modelo amplio, flexible y en continua mejora, que oriente el desarrollo académico y administrativo de toda la universidad, en sus diferentes sedes, dado por una visión sistémica de los procesos, con contextualización a las necesidades de desarrollo de las diferentes regiones del 
país. Para ello, se busca la implementación del modelo mediante proyectos que tengan impacto en las comunidades.

Atender en los espacios educativos y sociales la inclusión del desarrollo social sostenible, pretende redirigir el desarrollo de la sociedad moderna, mediante la transformación de las estrategias de desarrollo, tomando como base la sociedad del conocimiento mediante la articulación de forma estratégica entre la actividad que concibe el desarrollo social sostenible con el ámbito educativo y el principio de la socio formación, para aprender a resolver problemas con sentido de responsabilidad (Santoyo-Ledesma, 2019).

\section{CONCLUSIONES}

Es de vital importancia generar sensibilidad sobre el concepto de desarrollo sostenible para promover y potencializar cambios de actitudes necesarios para alcanzar impactos positivos en el entorno. Por tanto, respetar y cuidar la naturaleza se vuelve parte de dicha concientización para proveer de servicios a la humanidad mediante el aprovisionamiento y la regulación de recursos.

El desarrollo social sostenible, desde el punto de vista de la socioformación, busca identificar y establecer estrategias nuevas y mejoradas de aprendizaje social en los diferentes escenarios comunitarios, basado en diferentes ejes como: la inclusión social, el trabajo colaborativo con todos los actores que nos rodean, para mejorar de forma permanente las condiciones de vida y brindar una educación de calidad a los futuros profesionales.

Basados en la enseñanza integrada por conocimientos y métodos productivos que permita fomentar el desarrollo de la sociedad través de proyectos integrados a resolver problemas reales, se logrará la satisfacción de las necesidades básicas que preserven el ambiente.

El desarrollo social sostenible a partir de la gestión con enfoque socioformativo aborda ejes principales como es el proyecto ético de vida, la colaboración, el pensamiento complejo, la gestión del conocimiento y la metacognición y el compromiso social en pro del bienestar colectivo y del ambiente, con proyección hacia el futuro de forman que el cambio se torne tranversal en todas y cada una de las actividades universitarias.

\section{REFERENCIAS}

Ambrosio, R. (2018). La socioformación: un enfoque de cambio educativo. Revista Iberoamericana de Educación, 76, 57-82.

Arnao, M., Medina, K., Calderón, F., Esquivel, I. \& Tello, S. (2014). Socioformación y competencia comunicativa oral en Educación Secundaria. "UCV-HACER" Revista de investigación y cultura. 3 (2). Recuperado de http://www.redalyc.org/articulo.oa?id=521751976003 (26/09/2018)

Borrego Rodriguez, R. (2018). Desarrollo Sostenible desde la Socioformación. Memorias del II Congreso Internacional de Tecnología, Ciencia y. México.

Castro Acosta, A. (2018). Retos de la tecnología, la ciencia y la educación para contribuir a la salud y el bienestar sostenible. Colombia: CITED.

Coguox, A., Sandoval, L., \& Izquierdo, M. (2018). El Desarrollo Social Sostenible con enfoque Socioformativo a partir de la Gestión del Conocimiento. 
Memorias del II congreso Internacional de Tecnología, Ciencia y Educación para el Desarrollo Sostenible, (págs. 1-3). México.

Díaz-Barriga, F. (2010). Los profesores ante las innovaciones curriculares. Revista Iberoamericana de Educación Superior, 35-57.

Eastmond, A. (2005). La Sociedad del conocimiento, el desarrollo sustentable y ell papel de la educación superior en México en el fomento de la cultura ambiental. Revista de la Educación Superior Vol. XXXIV (4), No. 136, octubre-Diciembre de 2005, pp. 65-76. ISSN: 0185-2760, recuperado de http://publicaciones.anuies.mx/pdfs/revista/Revista136_S2A3ES.pdf.

Fernandez de Pelekais, C., \& D’Addosio Serna, R. (2013). Desarrollo social sostenible como resultado de la gerencia académica en las organizaciones universitarias. REDHECS, 1-5.

Fernández, L., \& Gutiérrez, M. (2013). Bienestar Social, Económico y Ambiental para las Presentes y. Información Tecnólogica.

Indoamérica, U. (2018). Modelo Educativo de la Universidad Indoamerica. Ambato, Tungurahua, Ecuador.

Jarrín, \& Tola. (2016). Lozano. (2018).

Juárez-Hernández, L., Tobón, S., Salas-Razo, G., \& Jerónimo-Cano, A. Y.-V. (2019). Desarrollo Sostenible: Educación y Sociedad. Revista Electrónic@ De Medio Ambiente.M

Kruger, K. (2006). El concepto de sociedad del conocimiento. Revista bibliográfica de Geografía y Ciencias Sociales. Obtenido de http://www.ub.edu/geocrit/b3w-683.htm

Martínez, J. E., Tobón, S., \& Ramírez, E. L. (2019). Currículo: un análisis desde un enfoque socioformativo. Revista de la invetsigación educativa, 43-63.

Martínez, T., \& Vasquez. (2017).

Moreno-Hernández, M., Rivera-Hernández, J., \& Rivera-Moreno, T. (2017). Competencias y aprendizajes del docente: Un reto de la globalización. Hexágono Pedagógico, 8(1), 180-197. https://doi.org/https://doi.org/10.22519/2145888X.1078

Narváez-Santos, L. J. (2018). Desarrollo social sostenible y socioformación. En J. Vélez Memorias del II Congreso Internacional de Tecnología, Ciencia y Educación para el Desarrollo Sostenible (CITED).

Niño-Castillo, J. \& Niño-Castillo, I. (2018). La Socioformación como herramienta factible en la educación actual. En L. G. Juárez-Hernández y S. Tobón (Coords.), Cuarto Congreso Internacional en Socioformación y Sociedad del Conocimiento. México: CIFE.

Rodríguez, E. (2009). El rol de las universidades en la sociedad del conocimiento y en la era de la globalización. . Revista Interciencia, 34.

Salud., O. P. (2013). Salud, ambiente y desarrollo sostenible: hacia el futuro que quremos. Una colección de textos basado en la serie de seminarios de la OPS hacia Rio+20.

Santoyo-Ledesma, D. S. (2019). Enfoque del Desarrollo Social Sostenible y la Gestión del Talento Humano en el contextode la Socioformación. Ecocience Internacional Journal, 8.

Slater, R. O. (2014). Educación en y para una sociedad del conocimiento. Educación, 14, 77-84. Stehr, N. (1994): Knowledge societies. The 
transformation of labour, property and knowledge in contemporary society. Londres: Sage.

Tahini, I., Nakayama, T., Dibrova, V. \& Dadykin, A. (2018). Cognitive psycology models and approaches to develop language skills. International Journal of Social Science and Humanity, Vol. 8, No. 4, April 2018. 110 doi: 10.18178/ijssh.2018.8.4.945 recuperado de http://www.ijssh.org/vol8/945SK209.pdf (26/09/2018).

Tedesco, J. C. (1999). Educación y sociedad del conocimiento y de la información. Memorias del Encuentro Internacional de Educación Media. Bogotá: Secretaría de Educación de Bogotá.

Tobón, S., Guzmán, C., Hernández, J. S., \& Cardona, S. (2015). Sociedad del conocimiento: Estudio documental desde una perspectiva humanista y compleja. Paradígma, 36(2), 7-36.

Tobón, S., Cardona, S., Vélez-Ramos, J., \& López-Loya, J. (2015). Proyectos formativos y desarrollo del talento humano para la sociedad del conocimiento. Revista Acción Pedagógica, No 24, enero - diciembre, 2015 - pp. 20 - 31.

Tobón, S. (2015). Cartografía conceptual: estrategia para la formación y evaluación de conceptos y teorías. CIFE.

Tobón, Pimienta, \& García. (2016).

Tobón, S. (2018). Es el desarrollo humano integral con acciones concretas de cuidado del ambiente mediante la colaboración. Colombia.

Tobón, S. (2018, noviembre). Proyectos formativos y habilidades socioemocionales: una estrategia para el desarrollo social sostenible. En R. Ordoñez (Moderador), II Congreso Internacional de Tecnología, Ciencia y Educación para el Desarrollo Sostenible (CITED-2018). Congreso conducido por el Centro Universitario CIFE, Cuernavaca, México (www.cife.edu.mx).

UNESCO. (2020). Unesco.org. Obtenido de https://es.unesco.org/themes/educacion-desarrollo-sostenible/comprenderEDS/decenio-onu.

Unidas, N. (2011). Panorama Social de America Latina. CEPAL, 150-151

Viglierchio, M.d.C., \& Williamson, D. M. (2015). Relación entre el concepto "sociedad del conocimiento" y la educación superior. Ciencia Veterinaria, 17 (1), 125-135.

Zabalza, M. Á. (2007). Competencias docentes del profesorado universitario. . Calidad y desarrollo profesional (2da. ed.). 